\title{
Can stem cell therapy increase the rate of myocardial recovery in left ventricular assist device-supported advanced heart failure patients? - current data and future perspectives
}

\author{
Gregor Poglajen ${ }^{1,2}$, Bojan Vrtovec ${ }^{1,2}$ \\ ${ }^{1}$ Advanced Heart Failure and Transplantation Center, Department of Cardiology, University Medical Center Ljubljana, Slovenia; ${ }^{2}$ Medical Faculty, \\ University of Ljubljana, Slovenia \\ Correspondence to: Gregor Poglajen, MD, PhD. Advanced Heart Failure and Transplantation Center, Department of Cardiology, University Medical \\ Center Ljubljana, Slovenia. Email: gregor.poglajen@kclj.si. \\ Provenance: This is an invited article commissioned by the Section Editor Dr. Liuhua Zhou (Department of Urology, Nanjing First Hospital, Nanjing \\ Medical University, Nanjing, China). \\ Comment on: Yau TM, Pagani FD, Mancini DM, et al. Intramyocardial injection of mesenchymal precursor cells and successful temporary weaning \\ from left ventricular assist device support in patients with advanced heart failure: a randomized clinical trial. JAMA 2019;321:1176-86.
}

Submitted Oct 01, 2019. Accepted for publication Oct 12, 2019.

doi: $10.21037 / \mathrm{atm} .2019 .10 .60$

View this article at: http://dx.doi.org/10.21037/atm.2019.10.60

Despite advances in medical management and left ventricular assist device (LVAD) technology, advanced chronic heart failure still represents a debilitating disorder with poor prognosis and high mortality rates (1). However, despite this, in selected patients with advanced chronic heart failure, myocardial structure and function can improve either spontaneusly or as a result of different therapeutic interventions. This improvement is believed to be achieved through a sophisticated interplay of cellular and subcellular changes affecting cardiomyocyte size, structure and function, as well as the structure and function of extracellular matrix (ECM). Together, these changes are commonly referred to as myocardial reverse remodeling and may, in a selected group of advanced chronic heart failure patients, result in improved structure and function of the failing myocardium. In terms of the magnitude of structural and functional restitution, reverse remodeling may be partial or complete, and in temporal terms, it may be permanent (myocardial recovery) or transient (myocardial remission) (2). Although reverse remodeling represents the main therapeutic target for all currently available and upcoming heart failure treatment modalities, the underlying pathophysiological mechanisms remain only partially understood. Specifically, a significant gap in knowledge exists regarding the association between structural and functional recovery of the failing heart. This disparity is most evident in LVAD-supported advanced chronic heart failure patients, where despite the reverse remodeling of the myocardial structure, only a small fraction (1-2\%) of patients achieve sufficient recovery of myocardial function to eventually undergo successful weaning and explantation of LVAD (3).

Recently, cell therapy has emerged as a promising treatment option for advanced chronic heart failure patients due to its potential to augment structural and functional reverse remodeling of the failing myocardium (4). However, it appears that this therapy may be less effective in patients with advanced heart failure and may not be sufficient to achieve stable myocardial recovery in this patient population $(5,6)$.

Theoretically, stem cell therapy and LVAD support should represent an ideal complementary treatment option for advanced chronic heart failure. Both cell therapy and LVAD support have been shown to beneficially affect the failing cardiomyocytes through hypertrophy reduction, improvement of metabolism and microvascular dysfunction. However, cell therapy and LVAD support act in opposite manner with regards to the myocardial tissue inflammation and remodeling of ECM: while LVAD therapy has been shown to have pro-fibrotic effects, stem cell therapy was associated with reduced fibrosis of $\operatorname{ECM}(7,8)$. On the other hand, mechanical unloading the failing myocardium could possibly decrease stem cell attrition rate after 
intramyocardial delivery by reducing left ventricular wall tension, improving myocardial perfusion and by creating a more favorable cytokine environment in the failing myocardium through a decrease in myocardial inflammatory response (9). This could result in an increased stem cell retention in the failing myocardium and, consequently, in more pronounced effects of cell therapy on angiogenesis and remodeling of ECM.

To date, data on the combined effects of LVAD and cell therapy are very scarce, despite the fact that transendocardial electromechanical mapping and stem cell injections were demonstrated to be feasible and safe in the presence of an LVAD support system (10). So far, only three studies investigated the LVAD/cell combination therapy in clinical settings. In a pilot trial, Ascheim and coworkers evaluated the safety and efficacy of allogeneic mesenchymal precursor cells (MPC) in 30 patients with ischemic and non-ischemic heart failure (11). At the time of LVAD implantation, patients were randomized in 2:1 manner to receive either transepicardial injections of mesenchymal precursor cells (MPC, $25 \times 10^{6}$ cells) or sham injection. The efficacy end points were patients' functional status and the function of left ventricle while temporarily weaned off LVAD support. The safety end points were the incidence of infectious myocarditis, myocardial rupture, neoplasm, hypersensitivity reaction, and allosensitization. The authors concluded that stem cell transplantation in this patient cohort was safe with regards to the procedure itself and potential allosensitization of the patient. They were also able to show a potential efficacy signal as patients in the MPC cell group had an increased likelihood of successful temporal LVAD weaning in comparison to controls (11). In a non-randomized study of Stampien-Otero and coworkers, 6 patients with ischemic heart failure received $0.5 \times 10^{6}$ CD $34+$ cells, $1.0 \times 10^{6} \mathrm{CD} 34-$ cells and $1.0 \times 10^{6}$ bone marrow mononuclear cells transepicardially in different parts of the left ventricular wall at the time of LVAD implantation (12). All hearts were histologically examined after heart transplantation and showed no significant differences in histological or inflammatory properties or in microvascular density between CD34+ cell-injected and saline-injected segments of the myocardium (12). The authors concluded that while in patients with ischemic heart failure undergoing LVAD implantation transepicardial cell injections in the failing myocardium appear to be safe, cell therapy does not appear to confer any additional clinical benefit in this patient population (12).

Recently, Pagani and coworkers reported the results of the largest cohort of patients receiving LVAD and cell therapy to date (13). In a multicenter study, 159 patients with ischemic and non-ischemic heart failure were randomized in 2:1 fashion to receive either transepicardial injections of allogeneic MPCs $\left(150 \times 10^{6}\right.$ cells $)$ or sham injections at the time of LVAD implantation (13). The primary efficacy end point of this study was the proportion of successful temporary weans (of 3 planned assessments) from LVAD support within 6 months. The 1-year primary safety end point was the incidence of intervention-related adverse events (myocarditis, myocardial rupture, neoplasm, hypersensitivity reactions, and immune sensitization). Secondary end points included readmissions and adverse events at 6 months, and 1-year survival (13). This study failed to meet its primary efficacy endpoint as 58 of 93 patients $(62 \%)$ in the study group vs. 26 of 42 control patients $(62 \%)$ were successfully temporarily weaned off LVAD support at 6 months (13). The mean proportion of successful weans over 6 months was $61 \%$ in the study group and $58 \%$ in the control group [risk ratio (RR)] for wean success rate, 1.08; 95\% CI, 0.83-1.41; $\mathrm{P}=0.55$ ) (13). In terms of secondary end points, the authors also failed to establish any differences between the two groups (13). Considering 1-year primary safety end points, transepicardial cell injections at the time of LVAD implant were shown to be safe and were not associated with any considerable side effects (13) which is in line with the data from the pilot trial (11). Importantly, this study again confirmed that the use of allogeneic cell product does not increase the rate of allosensitization in LVAD supported patients. However, the study did show, that patients in study group experienced significantly less mucosal bleeding (epistaxis and gastrointestinal) that the patients in control group. The authors speculated that MPC administration in study group may have resulted in higher systemic levels of angiopoetin-1, leading to reestablishment of the systemic balance of angiogenetic factors, and thus contributing to maintenance of vessel stability (13). Although this hypothesis is intriguing and may result in significant clinical benefits, it needs to be further explored through dedicated pre-clinical and clinical data.

Although these trials generated very important preliminary results, they do present several significant limitations. First, the low patient numbers in the trials preclude any definite conclusions regarding the efficacy of LVAD/cell combination therapy in advanced chronic heart failure patient cohort. Second, apart from the trial of Pagani and coworkers all studies used low numbers of 
stem cells in comparison to other positive clinical trials, performed in general heart failure population that usually used up to $50-100 \times 10^{6}$ stem cells per patient. Third, the most significant limitation of all three trials is the injection of cells transepicardially at the time of LVAD implantation. Although simultaneous LVAD and cell therapy appears more straightforward and feasible, this treatment strategy may significantly reduce the beneficial effects of this combined treatment approach as the local pro-inflammatory cytokine environment at the time of LVAD implantation (due to extracorporeal circulation and cardioplegic arrest of the myocardium) and "blind" transepicardial stem cell injections (as opposed to electroanatomically-guided cell injections) may adversely affect stem cell retention and survival.

Conversely, postponing stem cell therapy to the time when the patient has recovered from the LVAD implantation and the pro-inflammatory cytokine environment in the failing myocardium caused by LVAD surgery has abated may significantly increase the effects of the combinatory LVAD - stem cell treatment approach. However, in the case of delayed stem cell therapy, cells have to be delivered to the target myocardium transendocardially. Although this may seem more cumbersome, electromechanical mapping of the failing myocardium significantly improves the selection of optimal target areas for cell implantation. With this, electromechanically guided stem cell injections actually improve cell retention and survival (as they are not injected into the scar tissue) and may thus further increase the biological and clinical efficacy of cell therapy in this patient cohort (10).

Future trials exploring a combination of cell and LVAD therapy should meticulously and concisely address these issues and should be done in a standardized manner so the results could be adequately compared between the different trials and institutions. Additionally, future studies should focus not only on complete myocardial recovery and LVAD explantation, but also on the evaluation of the degree of reverse remodeling of the failing myocardium. Following standardized protocols may lead to a higher treatment success rate LVAD/cell combination therapy and could allow for better understanding of the biological processes associated with reverse remodeling and myocardial recovery in advanced chronic heart failure patients.

\section{Acknowledgments}

None.

\section{Footnote}

Conflicts of Interest: The authors have no conflicts of interest to declare.

Ethical Statement: The authors are accountable for all aspects of the work in ensuring that questions related to the accuracy or integrity of any part of the work are appropriately investigated and resolved.

\section{References}

1. Benjamin EJ, Blaha MJ, Chiuve SE, et al. Heart Disease and Stroke Statistics-2017 Update: A Report From the American Heart Association. Circulation 2017;135:e146-603.

2. Mann DL, Barger PM, Burkhoff D. Myocardial recovery and the failing heart: myth, magic, or molecular target? J Am Coll Cardiol 2012;60:2465-72.

3. Topkara VK, Garan AR, Fine B, et al. Myocardial Recovery in Patients Receiving Contemporary Left Ventricular Assist Devices: Results From the Interagency Registry for Mechanically Assisted Circulatory Support (INTERMACS). Circ Heart Fail 2016. doi: 10.1161/ CIRCHEARTFAILURE.116.003157.

4. Poglajen G, Vrtovec B. Stem cell therapy for chronic heart failure. Curr Opin Cardiol 2015;30:301-10.

5. Vrtovec B. Cell Therapy for Nonischemic Cardiomyopathy: Current Status and Future Perspectives. Circ Res 2018;122:28-30.

6. Vrtovec B, Poglajen G, Haddad F. Stem cell therapy in patients with heart failure. Methodist Debakey Cardiovasc J 2013;9:6-10.

7. Drakos SG, Kfoury AG, Hammond EH, et al. Impact of mechanical unloading on microvasculature and associated central remodeling features of the failing human heart. J Am Coll Cardiol 2010;56:382-91.

8. Malliaras K, Makkar RR, Smith RR, et al. Intracoronary cardiosphere-derived cells after myocardial infarction: evidence of therapeutic regeneration in the final 1-year results of the CADUCEUS trial (CArdiosphereDerived aUtologous stem CElls to reverse ventricUlar dySfunction). J Am Coll Cardiol 2014;63:110-22.

9. Hall JL, Fermin DR, Birks EJ, et al. Clinical, molecular, and genomic changes in response to a left ventricular assist device. J Am Coll Cardiol 2011;57:641-52.

10. Zheng Y, Sampaio LC, Li K, et al. Safety and feasibility of mapping and stem cell delivery in the presence of an implanted left ventricular assist device: a preclinical 
investigation in sheep. Tex Heart Inst J 2013;40:229-34.

11. Ascheim DD, Gelijns AC, Goldstein D, et al. Mesenchymal precursor cells as adjunctive therapy in recipients of contemporary left ventricular assist devices. Circulation 2014;129:2287-96.

12. Stempien-Otero A, Helterline D, Plummer T, et al. Mechanisms of bone marrow-derived cell therapy in ischemic cardiomyopathy with left ventricular assist device bridge to transplant. J Am Coll Cardiol 2015;65:1424-34.

13. Yau TM, Pagani FD, Mancini DM, et al. Intramyocardial injection of mesenchymal precursor cells and successful temporary weaning from left ventricular assist device support in patients with advanced heart failure: a randomized clinical trial. JAMA 2019;321:1176-86.

Cite this article as: Poglajen G, Vrtovec B. Can stem cell therapy increase the rate of myocardial recovery in left ventricular assist device-supported advanced heart failure patients? - current data and future perspectives. Ann Transl Med 2019;7(22):613. doi: 10.21037/atm.2019.10.60 